\title{
Cooperativity-Dependent Long-Lasting Potentiation in the Crayfish Lateral Giant Escape Reaction Circuit
}

\author{
Mark W. Miller, Sunhee C. Lee, and Franklin B. Krasne \\ Department of Psychology and Brain Research Institute, University of California, Los Angeles, Los Angeles, California \\ 90024
}

\begin{abstract}
The ability of sensory neuron firing to cause the lateral giant escape reaction increases following repeated sensory volleys at $4 \mathrm{~Hz}$ for $10 \mathrm{sec}$. The increase occurs only when relatively large numbers of afferents are repetitively stimulated, decays with a mean time constant of 21 hr, is confined to the ganglia at which the repeated sensory volleys enter the nerve cord, and is at least partially specific to those roots of a ganglion that were tetanized. Transmission at the chemical synapses between afferents and the largest of the first-order sensory neurons that link afferents to the lateral giants displays a similar potentiation. This phenomenon shares many properties with hippocampal long-term potentiation.
\end{abstract}

Bliss and Lomo reported in 1973 that high-frequency trains of action potentials in perforant path afferents increased efficacy of transmission to monosynaptic follower cells in the dentate gyrus of hippocampus for the duration of their acute experiments. This long-term potentiation (LTP) has now been found and studied at almost all of the major synaptic relays of the hippocampus, though all of the widely accepted characteristics of the phenomenon reviewed below may not be completely general. It is now known that LTP can last for hours and in at least some cases may last for weeks or longer (Bliss and GardnerMedwin, 1973; Douglas and Goddard, 1975; Racine et al., 1983). It has also been established that the potentiation is at least somewhat specific to synapses made by those afferents that were tetanized (i.e., it is "homosynaptic") (Anderson et al., 1977; Dunwiddie and Lynch, 1978); yet a relatively large set of afferents must be recruited during the tetanus to induce it (a phenomenon called "cooperativity") (McNaughton et al., 1978). Thus, production of potentiation at the synapse(s) made by a particular tetanically stimulated fiber depends on many other afferents being tetanized along with it. More recently, it has been shown explicitly that paired tetani of a "weak" test pathway (i.e., one in which not many fibers are fired) and a "strong" conditioning pathway (i.c., onc in which many fibers are fired) produced LTP in the weak pathway even though independent weak or strong tetani had no effect (Barrionuevo and Brown, 1983; Levy and Steward, 1983; Kelso and Brown, 1986). Thus, LTP has been called "associative," and the mechanisms un-

\footnotetext{
Received May 23, 1986; revised Oct. 28, 1986; accepted Nov. 10, 1986.

This work was supported by USPHS Grant NS 08108. We thank Paul Anders and Terri Teshiba for considerable help with chronic experiments.

Correspondence should be addressed to Frank Krasne at the above address.

Copyright (C) 1987 Society for Neuroscience $0270-6474 / 87 / 041081-12 \$ 02.00 / 0$
}

derlying it have come to be regarded as possibly related to the nervous system's capacity for associative learning.

Further investigation of these mechanisms has not yielded unequivocal conclusions. Thus, there is a variety of views as to the likely site of change [pre- or postsynaptic (see Bliss and Dolphin, 1984)], the nature of the change [augmented sensitivity to transmitters (I ynch and Baudry, 1984), altered membrane potential or conductance (Sastry, 1982), increased electrotonic spread due to altered morphology (Lee et al., 1980), or new synapses (Chang and Greenough, 1984)], the site of the convergence that is implied by cooperativity [the same postsynaptic neurons whose responses become potentiated (Lynch and Baudry, 1984) or independent neurons whose input to the neurons of potentiated pathways promotes potentiation (Hopkins and Johnston, 1984)], and the nature of the signal by which strong inputs promote targets to change [elevated internal $\mathrm{Ca}^{2+}$ promoted by extreme depolarization (Lynch and Baudry, 1984) or receipt of special modulatory factors (see Collingridge, 1985)].

Some of these uncertainties may result from variations in methodology or in the particular hippocampal synapses chosen for analysis, and to the extent that this is the case, a concentration of experimental effort on hippocampus itself is obviously desirable. But in the hope of finding LTP in preparations offering special experimental advantages and of aiding separation of the essential from the fortuitous, LTP has been sought elsewhere. As a result, relatively long-lasting increases of synaptic efficacy that might be homologous to LTP have been uncovered at places as diverse as mammalian sympathetic ganglia (Briggs et al., 1985), crayfish neuromuscular junctions (Baxter et al., 1985), and synapses mediating the defensive gill and siphon withdrawal reflex of Aplysia (Walters and Byrne, 1985). At least in the short run this has not clarified the picture. At a time when there is an increasing consensus that postsynaptic change triggered by glutamatergic input to heavily depolarized target cells is an important contributor to hippocampal LTP (see Collingridge, 1985; Kelso et al., 1986; Malinow and Miller, 1986), several invertebrate preparations have contributed convincing evidence of a presynaptic locus of change and, in Aplysia, of a crucial role for aminergic or peptidergic modulators in producing LTP-like changes (Hawkins et al., 1983; Baxter et al., 1985; Walters and Byrne, 1985). Given this, further preparations are needed to determine whether we are dealing with vertebrate-invertebrate differences or simply with a range of mechanisms that may be available in many phyla. Also desirable are preparations in which the behavioral consequences of altered synaptic function are apparent, for such preparations will help us understand the biological functions of the phenomena uncovered. 

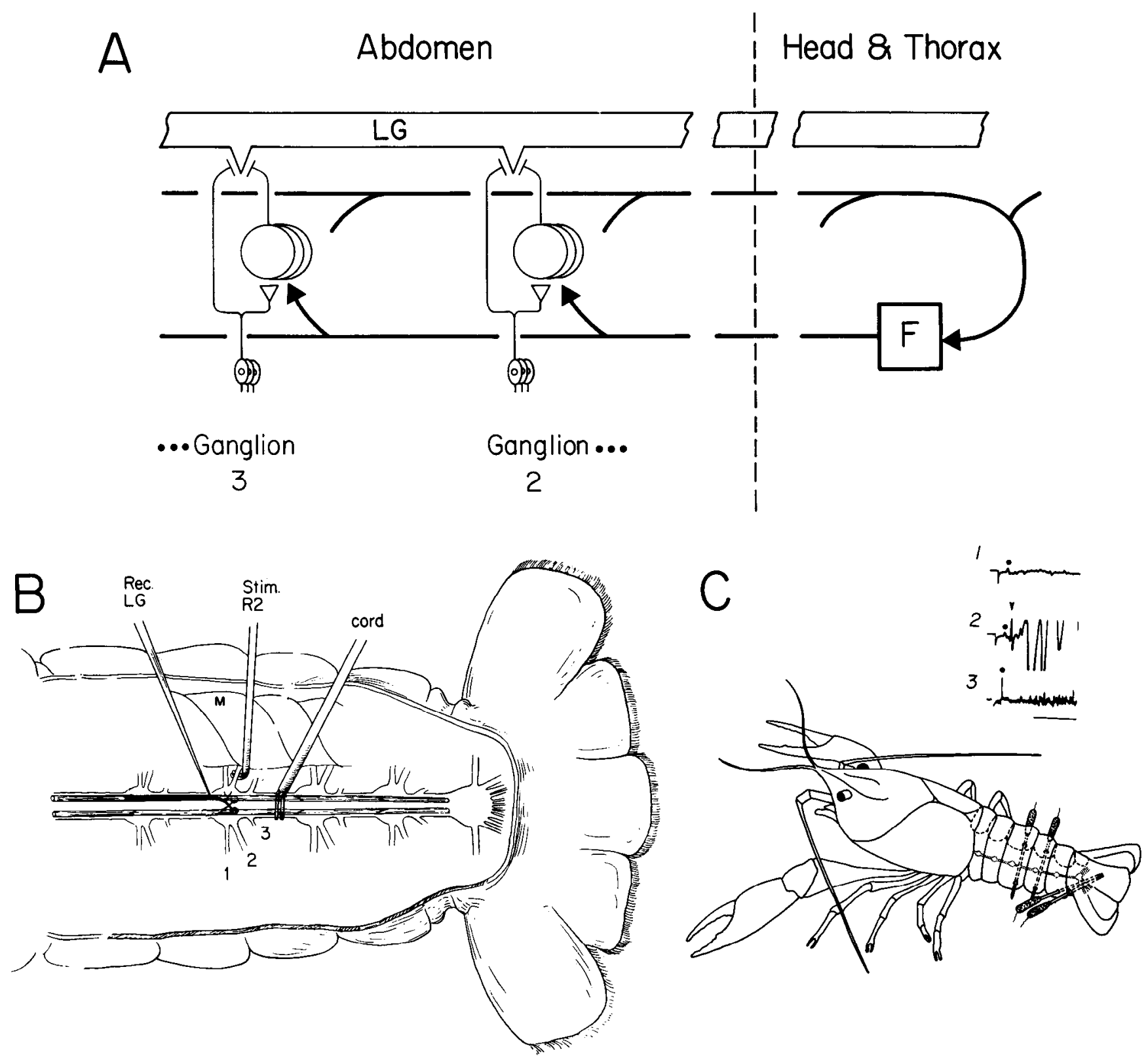

Figure 1. Escape reflex circuitry and experimental preparations. A, Segmentally repeated circuitry providing input to the LGs. Bold lines show the facilitatory system $(F)$ responsible for sensitizing the escape reflex; activity of the system is projected throughout the nervous system after having been recruited rostrally. $B$, Dorsal schematic view of an acute preparation. Roots 1-3 are numbered in the 3rd abdominal ganglion. Recording electrodes are at the dendritic end of the segment of the LG that receives input in the 3rd abdominal ganglion. LG segments communicate via bidirectional electrical synapses with a high safety factor so that the joined LG axon segments act in effect as a single axon. $C$, Chronically implanted preparation showing recording electrodes (rostral pair) and stimulating electrodes (caudal pair). Recording electrodes placed dorsal to the cord record LGs maximally, while electrodes placed ventrally record interneuron A maximally. At right are sample interneuron A spikes (dots) and LG spikes (arrowhead); 1 and 2 were recorded by a dorsal and 3 by a ventral electrode. Time calibration, $5 \mathrm{msec}$.

We report here the discovery of a cooperative LTP-like phenomenon in the circuit for lateral giant (LG) tailflip escape responses of crayfish. The primary afferents of the LG escape reaction are mechanoreceptors distributed over the dorsal and lateral surface of the 6 abdominal scgments and the terminal tailfan. They excite the LG dendrites in their ganglion of entry both monosynaptically and via a tier of interneurons, the excitation contributed to the LGs by the disynaptic pathway being about twice that from the monosynaptic one (which is alone usually unable to excite the LGs to firing threshold) (Fig. 1A). The synapses between primary afferents and interneurons are chemical, while both primary afferent and interneuron synapses onto the LGs are believed to be electrical (see Wine and Krasne, 1982). In the present experiments we sought evidence of LTPlike phenomena by examining the effects of trains of action potentials in sensory neurons on subsequent efficacy of the synapses in the circuit exciting the LGs.

The efficacy of the first (chemical) synapse, which is the one that seems most likely to be subject to change, is known to be modulated by a facilitatory pathway that becomes active during strong stimulation (prolonged AC shocks) of the head, the contralateral tailfan, and presumably also other bodily sites, pro- 

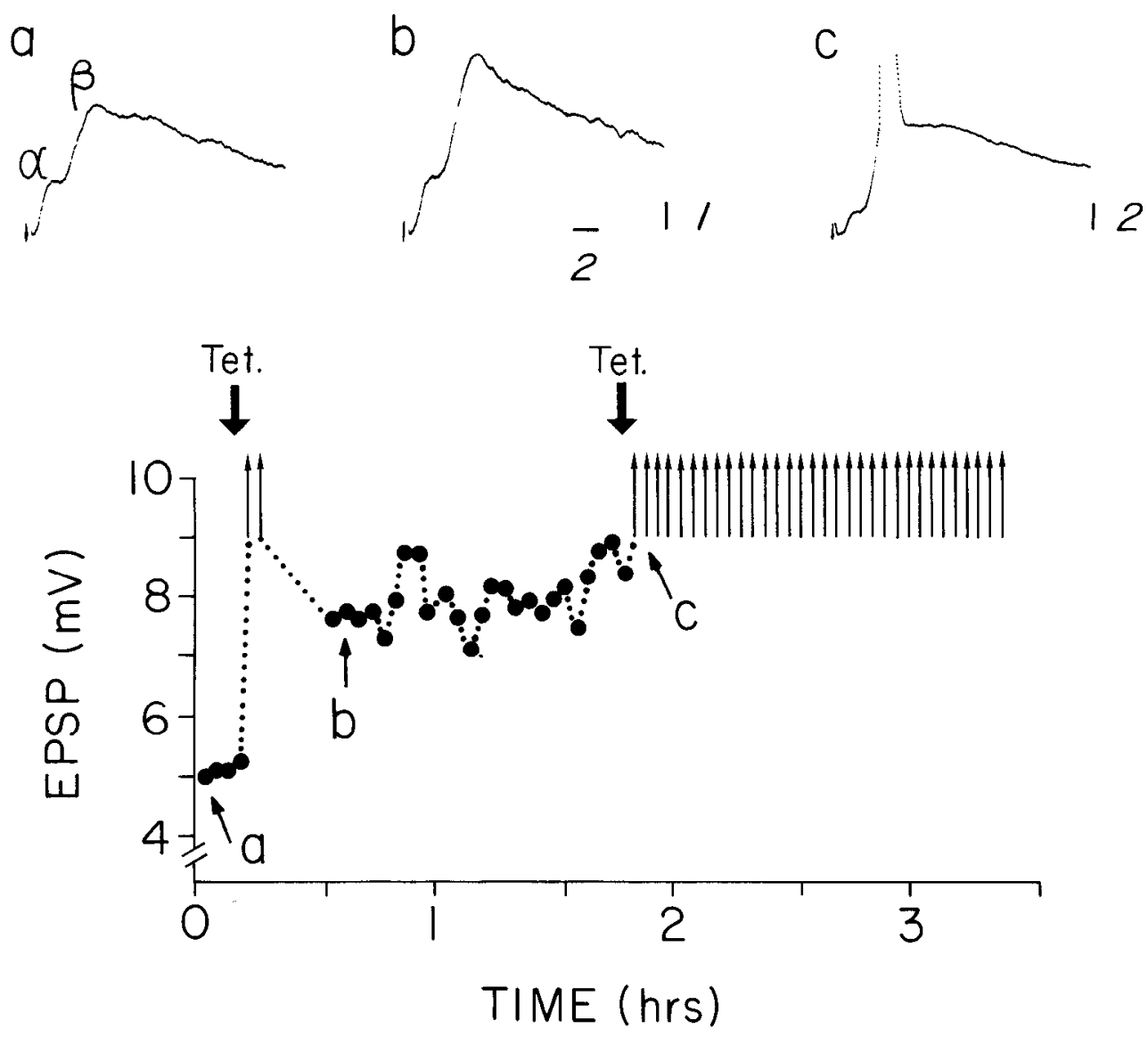

Figure 2. Long-term potentiation in an acute preparation. Test stimuli were given continuously at $3 \mathrm{~min}$ intervals. Tet. indicates a $10 \mathrm{sec}$ train of $4 \mathrm{~Hz}$ pulses of the same intensity as the test stimulus applied midway between 2 test pulses. EPSP amplitudes were measured to the peak of the beta component (see Materials and Methods). EPSPs greater than about $9 \mathrm{mV}$ triggered action potentials, which in the graph are indicated by upward arrows. Sample traces $a-c$ (above) were taken at the points indicated in the graph below. In $a$, the monosynaptic "alpha" and disynaptic "beta" components are indicated. Calibrations indicated are in $\mathrm{mV}$ and msec.

ducing a sensitization of the escape reaction (Krasne and Glanzman, 1986). Because strong stimuli, whether rostral or caudal, lose their ability to facilitate caudal targets after severance of the nerve cord between thorax and abdomen, input to the facilitatory system is believed to occur only rostral to the abdomen. Our search for LTP in the abdominal cord was, thus, conducted on animals with cords transected to prevent recruitment of the facilitatory system.

\section{Materials and Methods}

Animals. Procambarus clarkii 7-9 cm rostrum-to-telson were used in all experiments. They were obtained from various California suppliers.

Experimental design. In all experiments $0.1 \mathrm{msec}$ test shocks to sensory roots were delivered every $3 \mathrm{~min}$ (or occasionally every $2 \mathrm{~min}$ ). Low-frequency tetani, usually 10 -sec-long trains of $0.1 \mathrm{msec}$ pulses at $4 \mathrm{~Hz}$ were interposed as necessary halfway between one test shock and the next.

Acute preparations and testing. The nerve cords of animals cooled to about $5^{\circ} \mathrm{C}$ were severed at the connective just rostral to the last thoracic ganglion and the abdomen cut off and pinned dorsal side up in a dish filled with cold van Harreveld's solution (van Harreveld, 1936). The abdominal nerve cord was exposed dorsally by removing the exoskeleton and separating phasic flexor musculature; thereafter, a stream of well aerated $17^{\circ} \mathrm{C}$ van Harreveld's solution was continually flowed over the cord; in some experiments, $10 \mathrm{~mm}$ glucose was added. Pairs of platinum wire hook electrodes for stimulating were placed on the second roots of one or more of ganglia $2-5$, with the cathode about a cord width from the ganglion and the ipsilateral $L G$ impaled with $10-30 \mathrm{M} \Omega \mathrm{KCl}-$ filled microelectrodes in one of the ganglia having a second root stimulating electrode (Fig. 1 $B$; details are all as in Krasne, 1969).

Synaptic potentials evoked in the LG by second root shocks have an "alpha" component peaking at about $1.5 \mathrm{msec}$, reflecting input arriving directly from afferents via electrical synapses, and a larger "beta" component peaking at about $4 \mathrm{msec}$, reflecting input from first-order sensory interneurons that receive chemical synapses from primary afferents and that transmit electrically to the LGs (see Fig. 2a). At the start of acute experiments the test stimulus voltage was adjusted to give a beta component amplitude of approximately half the critical firing level of the LG. Unless otherwise stated, all LG EPSP amplitudes were measured at the peak of the beta component.

Chronic preparations and testing. Several days prior to initiation of experiments, nerve cords were severed between the first and second abdominal ganglion, a bipolar pair of stimulating "skewer" electrodes (see Krasne and Glanzman, 1986) chronically implanted over roots 2 4 of the last abdominal ganglion, and recording skewer electrodes implanted over either the giant axons or interneuron $A$, the largest of the first-order sensory interneurons of the $\mathrm{LG}$ circuit (Fig. $1 C$; details as in Krasne and Glanzman, 1986). In experiments used to assess homosynapticity, 1 monopolar skewer electrode was implanted over roots 2-4 and another over root 1; for each, the circuit was completed by an aquarium electrode; the large stimulation artifacts resulting from such monopolar stimulation were reduced by a high pass filter. Animals were maintained in individual 5 gal aquaria and their electrode leads run to floats at which connections to equipment were made a half-hour before experimental sessions.

During experimental sessions the amplitude of test shocks (given at 2 or 3 min intervals) was varied from trial to trial so as to maintain a running estimate of the stimulus threshold of the unit (LG or interneuron A) under observation (if a response did not occur on a test trial, the stimulus was increased on the next test trial, and conversely); this procedure (see further below) is referred to as "threshold tracking." Identification of units was as previously described (Krasne and Glanzman, 1986).

\section{Results}

Low-frequency tetani can produce persistent increases of efficacy

Acute preparations

It is known that repetitive activation of $L G$ reflex afferents at stimulus frequencies of from $1 / 2 \mathrm{sec}$ to $1 / 5 \mathrm{~min}$ can cause re- 


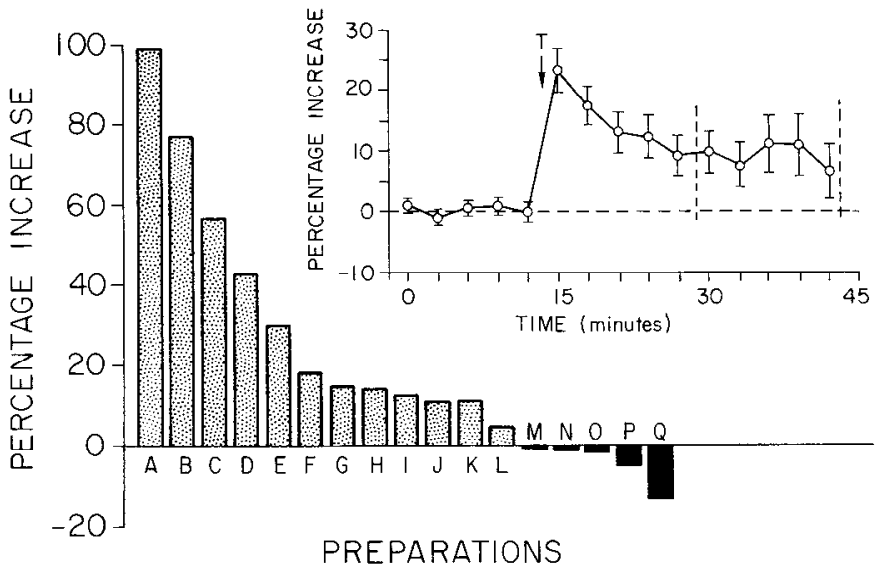

Figure 3. Effect of tetani in the first 17 acute preparations tested (labeled $A-Q$ ) and ordered by magnitude of potentiation. Mean percentage increases in EPSP amplitude relative to the 5 test trials preceding tetanus were computed for posttetanus trials 6-10 (16.5-31.5 min after tetanus); critical firing levels were entered in the analysis when, following tetani, spikes prevented measuring the full amplitude of the EPSP. For this analysis (and no other), the mean EPSPs for trials 6-10 were corrected for drift, assumed to be constant and linear, which was estimated from successive blocks of 5 trials prior to tetanus. In cases in which effects of more than a single tetanus were examined, averaged increases are presented. Inset, Trial-by-trial plot averaged across preparations. Preparations showing spiking were not included in this curve, since EPSP amplitudes could not be estimated when spiking occurred and the convention of entering firing levels might have distorted the time course. Error bars are SEMs. Vertical dashed lines indicate time period from which data of main figure were obtained.

duced excitation of the LGs ("depression"), which can persist, at least under certain conditions, for some hours (Wine et al., 1975). Here, our intention was to examine the effects of higherfrequency activation, and we anticipated that any potentiation occurring might be manifested only as diminished depression following high-frequency afferent volleys.

Our initial experiments informally explored tetani having pulse frequencies ranging from 50 to $4 \mathrm{~Hz}$ and durations from 1 to $10 \mathrm{sec}$, the amplitude of pulses during the train being the same as that of test pulses. All such tetani caused depression of the beta component during the train, and during the higher-frequency trains it was virtually abolished. However, at the first test shock, $90 \mathrm{sec}$ after the tetanus, responses were commonly larger than those prior to the train. The degree of potentiation was sometimes substantial but was highly variable. Although a systematic study of train parameters has yet to be done, it was clear that lower-frequency trains were not conspicuously less effective than those of higher frequency, and because inhibitory aftereffects of root shocks may last in excess of $100 \mathrm{msec}$ (Kennedy et al., 1974; Bryan and Krasne, 1977), we opted for the use of trains in which interpulse intervals were relatively long to avoid potentially complicating effects of such inhibition. Thus, in the bulk of later experiments we utilized a $4 \mathrm{~Hz}, 10$-sec-long train of pulses. Although in our initial experiments stimulus voltages during tetani were the same as those during testing, it gradually became apparent (see below) that better potentiation was produced when higher voltage stimulus pulses that recruited more afferents were used during tetani; hence, most experiments reported here utilized "strong" tetani (i.e., tetani of relatively high voltage pulses).

Figure 2 illustrates the results of the first experiment that examined the effect of $4 \mathrm{~Hz}, 10 \mathrm{sec}$ trains; potentiation was obviously large and prolonged. In this, as in most experiments, cnhancement was greatest at the first test, $90 \mathrm{sec}$ after the tetanus, and declined over some $5-10 \mathrm{~min}$ to a relative plateau from which further decline was much less rapid (see Fig. 3, inset). In some experiments, potentiation was seen only during the first 5-10 min following the tetanus. Because we were interested here primarily in relatively prolonged effects, most of our analyses are on effects that persisted beyond the initial period of relatively rapid decline.

Low-frequency tetani consisting of $4 \mathrm{~Hz}, 10 \mathrm{sec}$ long trains were initially employed in 17 preparations. Figure 3 presents an estimate of potentiation during the period 15-30 min posttetanus in each of them. Most of these were "strong" tetani in which the pulse voltage during the tetanus was twice that used for testing. However, in a few cases (13\%) in which "weak" tetani (tetanus pulse voltage equal to test pulse voltage) had previously caused potentiation in a preparation, the effects of a second weak tetanus were examined and the results (whatever the outcome) included in the analysis. For each preparation the mean of the 5 test EPSPs measured during minutes $15-30$ posttetanus and of the last 5 test EPSPs preceding the tetanus were used to calculate a percentage increase in EPSP amplitude. Since in some preparations the baseline EPSP showed noticeable drift prior to presentation of the tetanus (in 20\% it changed more than $10 \%$ in $30 \mathrm{~min}$ ), the percentage increases were corrected for drift under the working assumption that it was continuous and constant; although this assumption is surely not strictly correct, it should not have introduced any systematic bias into the data. It is apparent from Figure 3 that potentiation lasting more than 15 min occurred in about $60-70 \%$ of the preparations. The overall increase (including preparations that showed no potentiation) is statistically significant at $p<0.01$ (2-tailed Wilcoxon test on percentage increases).

The possibility that this potentiation was due to a posttetanus increase in the afferent volley seemed unlikely given the effect's longevity and the modest nature of the tetani. However, to check this more directly, we utilized the monosynaptic electrical synapse-mediated alpha component to assess constancy of the afferent root volley. Test shocks that produced a distinctly submaximal alpha component (and a negligible beta component) wcre altcrnated with larger test shocks that produced a maximal alpha component as well as a moderate beta component. In such preparations tetani could cause substantial potentiation of the beta component (evoked by the stronger test shock) without an increase of the alpha component (produced by either strength test shocks). We conclude that the effects are not due to afferent volley changes.

However, although potentiation of the beta component can occur without potentiation of the alpha component, in some preparations there was a small increase of the alpha component that paralleled that of the beta component (see Discussion).

\section{Chronic preparations}

In the hope of obtaining potentiation under conditions that would better allow assessment of the effect's longevity, we performed similar experiments on freely behaving chronic preparations. Figure $4 A$ shows a typical experiment. On a first, control day, LG firing threshold was tracked (see Materials and Methods) throughout a 2-3 hr session to evaluate the effects on excitability of repeated testing alone. In some animals, such as the one from which the data of Figure $4 A$ were taken, there was 


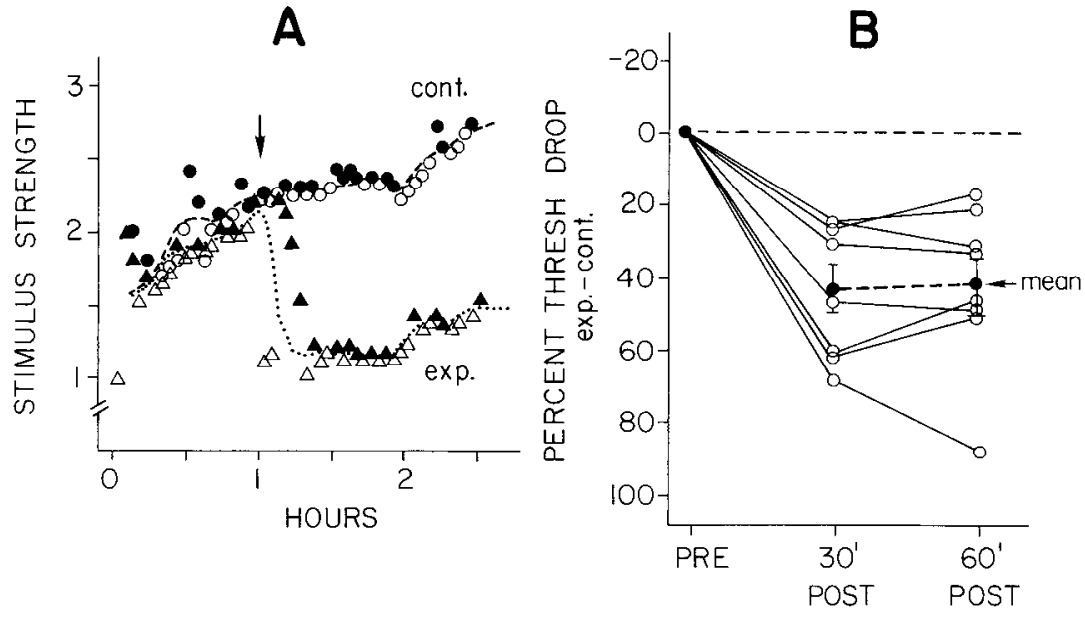

Figure 4. Potentiation in chronic preparations. $A$, Each point plots the time and stimulus strength for a test trial. Filled symbols indicate a response to the stimulus and open symbols a failure to respond. Results for a control session (circles) and an experimental session (triangles) are superimposed. The arrow indicates time of applying a strong tetanus in the experimental session. The dotted and dashed lines are subjective interpretations of threshold. $B$, Percentage threshold drops (see text) at 30 and 60 min posttetanus for the first 8 animals run in experiments comparable to that illustrated in $A$. Error bars on mean curve are SEMs. some habituation of reflex excitabilty throughout a session, while in other animals excitability was quite constant; threshold drops during control sessions were very rare. An experimental session was run the day after the control session. During experimental sessions, threshold was tracked for about $1 \mathrm{hr}$ prior to the delivery of a $10 \mathrm{sec}$ train of $4 \mathrm{~Hz}$ pulses at the midpoint of an intertest-pulse interval. The voltage pulses during these tetani were substantially suprathreshold, typically being sufficiently intense so that cach pulsc of the train evoked firing of the LGs. Threshold often dropped following the tetani, as in Figure $4 A$, below its pretetanus value; however, in a number of animals that habituated during test sessions, the tetanus merely served to partially counter habituation without actually causing an absolute drop. Because of this, for all animals we calculated percentage threshold drops for both experimental sessions and corresponding time points in control sessions and used the difference between the experimental and control session percentage drops as an index of potentiation. This difference gave a measure of potentiation from which effects of within-session habituation were largely factored out. Such differences at 30 and $60 \mathrm{~min}$ following tetani are given in Figure $4 B$ for 8 animals tested in this way. Averaging the 30 and $60 \mathrm{~min}$ point for each animal, the increase is highly significant ( $p<0.01,2$-tailed Wilcoxon test).

\section{Longevity of potentiation}

\section{Acute experiments}

The average time course of potentiation, starting at the first test $90 \mathrm{sec}$ after the tetanus and continuing for about half an hour, is shown in the inset of Figure 3 for a large number of experiments. Potentiation was maximal at the first test and decayed thereafter with at least two time constants. The time constant for the initial rate of fall was about $11 \mathrm{~min}$. The subsequent rate of decline was-considerably slower but difficult to estimate because the values are somewhat variable and the time period over which data was collected short compared to the duration of decay. Depending on how one draws single exponentials through the points, time constants ranging from $35 \mathrm{~min}$ to more than $24 \mathrm{hr}$ can be obtained. Evaluation of a few individual preparations in which there are somewhat longer periods of observation available indicate considerable interpreparation variability. Thus, in the preparation shown in Figure 2 there was no drop (indeed a slight rise) over more than an hour of observation, while in the preparation of Figure 5 potentiation decayed almost completely during the first hour after the tetanus (time constant being approximately $18 \mathrm{~min}$ estimated over $15-$ 45 min posttetanus).

\section{Chronic preparations}

In chronic preparations it is difficult to get a clear idea of the time course of potentiation immediately after the tetanus, because with the onset of potentiation one loses track of the threshold, and it takes some trials of tracking to relocate it; therefore, we did not attempt to estimate the initial rate of decay of potentiation in these preparations. An estimate of decay rate during the second half-hour after tetani was obtained from the average percentage threshold drop data of the 8 animals in Figure $4 B$. This gives a time constant of about $21 \mathrm{hr}$. Thus, although the potentiation is rather long lasting, it does not appear to be "permanent" as has been proposed for some hippocampal LTP. Consistent with this conclusion, there has been no indication

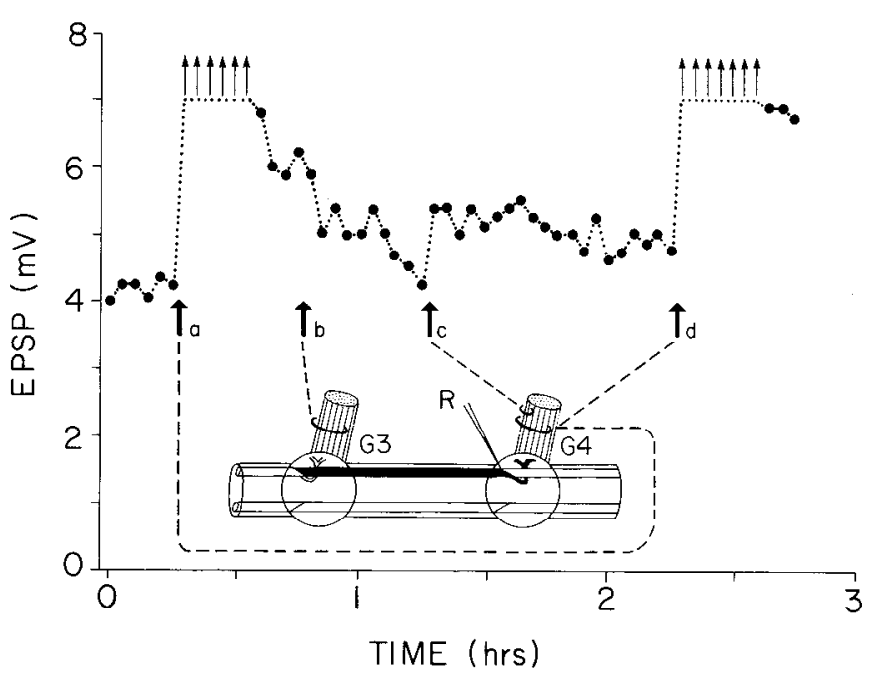

Figure 5. Effect of stimulus strength during tetanus and lack of transfer of potentiation between segments in acute preparations. Throughout the experiment "weak" test stimuli were delivered every $3 \mathrm{~min}$ to the 2nd root of the 4th abdominal ganglion and the resultant EPSP at the base of the ipsilateral LG dendrite in the same ganglion recorded. At $a$ and $d$ " "strong" tetani (pulse amplitude twice test amplitude) were delivered through the same root electrodes used for testing. Both such tetani caused potentiation. At $b$, a "strong" tetanus (same voltage as at $a$ and $d$ ) was delivered to the 2 nd root of the 3 rd abdominal ganglion with little or no effect. At $c$, a "weak" tetanus (stimulus intensity same as used during testing) caused at most a slight potentiation. 


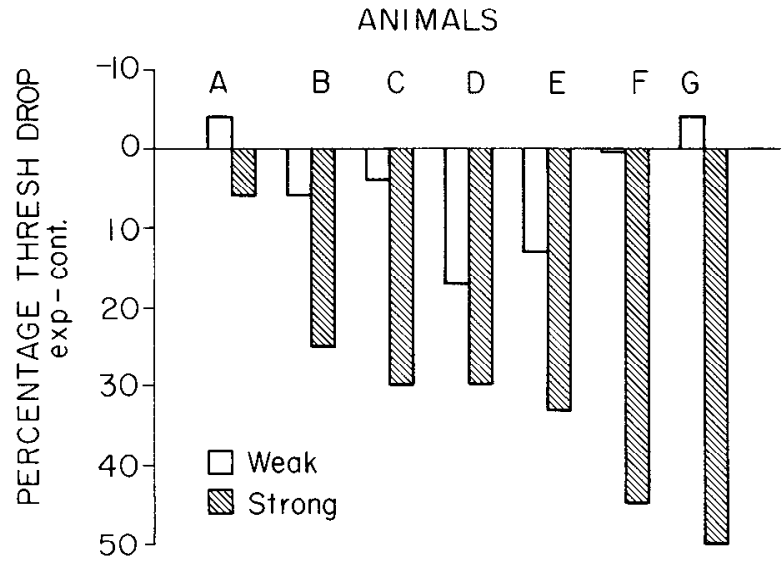

Figure 6. Cooperativity in chronic preparations. Each animal $(A-G)$ underwent 2 experimental sessions similar to that illustrated in Figure 4. For 1 session ("weak") stimulus strength during tetanus was just suprathreshold for LG firing prior to the train. For the other session ("strong") stimulus strength during tetanus was sufficiently strong to fire the LGs during every stimulus of the train. "Strong" was first for $A, C$, and $D$. The animals have been ordered according to degree of potentiation caused by strong tetani.

of persisting potentiation from one day to the next in animals tested over several sessions.

\section{Cooperativity}

Dependence on a substantial afferent volley during tetanus, i.e., cooperativity, is a hallmark of hippocampal LTP (see introduction). Figure 5 illustrates one of a number of acute experiments suggesting that the potentiation under examination here was also cooperativity-dependent. In this experiment, tetani in which the individual pulses were twice as strong as those used during testing ("strong" tetani, a and d) produced substantial potentiation at the start and again at the end of the experiment, whereas a tetanus in the middle of the experiment in which the pulses were at the same voltage used for testing (i.e., a "weak" tetanus, c) caused at most a small potentiation. For 9 acute experiments in which both "weak" and "strong" tetani (defined as above) were given, mean percentage increases in EPSP amplitude during the 15-30 min period after the tetani were significantly greater for the strong than the weak tetani [15.3 (SEM 5.6) vs 3.38 (SEM 2.3); $p<0.05$, 2-tailed Wilcoxon test]. It should be noted that mean percentage potentiation was negligible even during the first $15 \mathrm{~min}$ after weak tetani; thus, cooperativity probably applies to the rapidly, as well as the slowly, decaying component of potentiation in this circuit.

In chronic preparations, cooperativity was evaluated by comparing the potentiation produced by "weak" tetani, in which the stimulus pulse voltage was set to a level just suprathreshold for LG firing at the time the tetanus was begun, and "strong" tetani, in which the pulse voltage was great enough to cause LG firing on every pulse of the train (a few animals in which this could not be achieved were discarded). The differences between experimental and control threshold drops for such weak and strong tetani in each animal run are shown in Figure 6. In every animal more potentiation (a greater positive difference) occurred for strong than wcak trains $(p<0.02,2$-tailcd Wilcoxon test).

\section{Segmental specificity}

Because the present experiments were all done in preparations with abdominal nerve cords severed rostrally to isolate them from rostrally originating facilitatory influences, the potentiations observed are believed to depend upon the tetanic activity of neurons in the test pathway itself, along with that of the additional parallel neurons recruited by strong tetani. However, in order to confirm our belief that the tetani used here were not somehow recruiting activity of facilitatory neurons that mediate sensitization (which is produced even in segments very distal from those where the traumatic stimuli that cause it are applied), we examined the extent to which potentiation could be produced in our preparations in segments other than those tetanized. Figure 5 illustrates the format of an experiment. The LGs were impaled for recording in 1 segment and stimulating electrodes placed on ipsilateral second roots of this same segment (providing a "homosegmental" input pathway) as well as on the second root of an adjacent segment (providing a "heterosegmental" input pathway). All test stimuli were delivered homosegmentally, and effects of strong homosegmental (a and d) and heterosegmental (b) tetani (twice test-pulse amplitude) were each evaluated and compared. During both homo- and heterosynaptic tetani, the LGs fired during the first several pulses and failed thereafter; by the end of the tetani, beta components were considerably reduced but not completely gone. In the experiment shown, homosegmental tetani were obviously very effective, whereas otherwise comparable heterosegmental tetani were apparently without effect. Comparable experiments were performed in 9 preparations; EPSP increments, as a percentage of the mean of the 5 trials preceding the tetanus, are shown in Figure 7, with trial-by-trial means shown in the inset. It is obvious that heterosegmental tetani were without effect [unless it was a depressive one-reduction of test EPSPs under somewhat comparable circumstances have been reported in the hippocampus (see Swanson et al., 1982), but here the decline might equally be due to gradual habituation to test stimuli]. A statistical comparison between the average percentage change on trials 4-8 posttetanus produced by homo- and heterosegmental tetani (averaging the results of multiple homosegmental tests on a preparation) is significant at $p<0.01$ (2-tailed Wilcoxon test).

\section{Reliability of potentiation}

Despite some variation, potentiation in chronic preparations has been very reliable. Combining experiments on the LG and interneuron A (see below), the effects of strong trains have been evaluated in 23 preparations. In all of these a positive difference in threshold drop of experimental and control animals was obtained, and in $87 \%$ the difference was greater than $10 \%$.

By contrast, potentiation in acute experiments has been capricious. In our first series of acute experiments that used strong $4 \mathrm{~Hz}, 10 \mathrm{sec}$ tetani (the data of Fig. 3), which was run between January and April 1984, potentiation occurred in $60-70 \%$ of the preparations. However, in subsequent experiments during May-September, aimed at investigating some pharmacological manipulations, there was virtually no sign of potentiation. Starting in October the effect appeared to return. In 19 experiments run during November and December (the time of writing) potentiation has occurred in $60-70 \%$ of the preparations.

Thus at the present time it appears that in acute preparations the potentiation might be seasonal (occurring in the late fall, winter, and early spring but not the late spring and summer). By contrast, the effect in chronic preparations has been quite reliable, does not appear seasonal, and, in particular, was strong during the summer of 1985 , when virtually no potentiation could be obtained in acute preparations. 


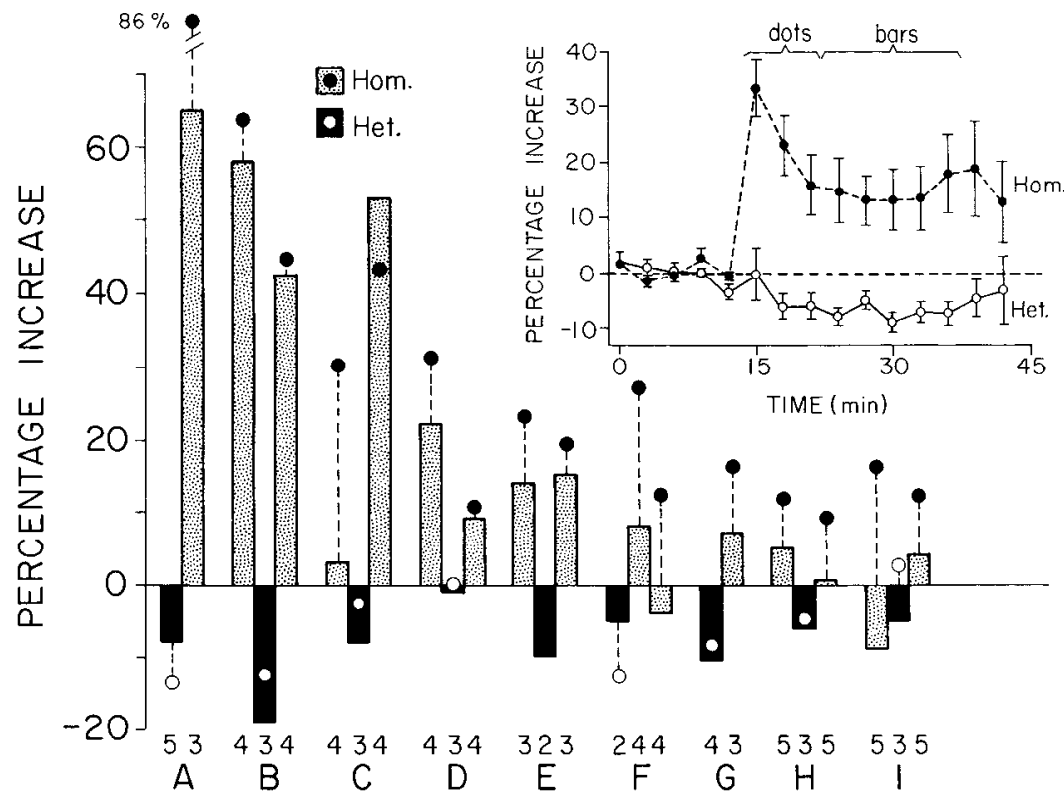

Figure 7. Potentiation is confined to the tetanized segmental ganglion. The experimental format was similar to that shown in Figure 5 (configurations $a$ and $b$-see text). Inset, Mean percentage increases ( \pm SEMs) plotted trial by trial for homo- and heterosegmental strong tetani (data from a few experimental sessions in which the full amplitude of EPSPs could not be measured due to spiking omitted). Brackets indicate the trials over which EPSPs were averaged for the bar graph. Bar graph, Mean increase on trials 1-3 posttetanus (dots) and 4-9 (bars) for each preparation (A-I); shaded bars and solid dots indicate homosegmental tetani, and black bars and open dots indicate heterosegmental tetani. Preparations were ranked roughly according to degree of potentiation shown. All sessions are included; in case of spiking, the critical firing level was entered as the EPSP amplitude. The order of presentation of bars for a preparation indicate the order in which the tetani were given; below each bar the ganglion tetanized is indicated. The preparations shown here are a subset of those in Figure 3.

The reasons for the unreliability of acute preparations and the greater reliability of chronic preparations are not resolved. Various possibilities present themselves (see Discussion); however, 2 clear differences between the acute and chronic experiments deserve mention here: (1) For reasons of technical convenience our acute experiments were done on abdominal ganglia 2-5, whereas all chronic experiments were done on ganglion 6 (the last abdominal). (2) Afferent volleys during tetani were more effective in driving the LG (and presumably the interneurons that preceed it) in chronic than in acute experiments. Thus, the LGs fired only to the first few afferent shocks to strong tetani in acute experiments, whereas they fired to every shock of strong trains in chronic preparations. In the light of these differences we performed a number of acute experiments on the last abdominal ganglion during March-June 1984. As in the acute experiments on more rostral ganglia, we were unable to produce LG spikes on more than the first few stimuli of a tetanus even with stimulus voltages substantially higher than those used in chronic experiments. Potentiation was also similar to that in more rostral ganglia. During March and April, when potentiation in rostral ganglia was common, it was also common in the last ganglion; during May and June when it was not occurring rostrally, it also failed to occur caudally. Thus, the differences between the acute and chronic experiments do not appear to be related to ganglionic differences per se. The possibility remains that they are due to the greater efficacy of the afferent volley in the chronic preparations.

Potentiation at the first, chemical synapse of the LG circuit

Because chemical synapses have more commonly been found to be plastic than electrical ones (but see further in Discussion), we expected the synapse between primary afferents and sensory interneurons to be the major locus of potentiation. To evaluate the presence of potentiation at this level, we examined transmission between primary afferents and interneuron $A$ (hereafter $A$ ), the largest of the sensory interneurons in the cord. The experiments, performed on chronic preparations, were essentially the same in format as those on the LGs in Figure 4 except that chronic recording electrodes were used to record interneuron $\mathrm{A}$ rather than $\mathrm{LG}$ firing. Figure $8 A$ illustrates an experiment in which we examined the effects of both weak and strong tetani on the sensory threshold for firing $A$. On a control day, the excitability of $A$ was stable. On a subsequent experimental day, threshold was slightly lower (perhaps due to a shift of stimulating electrode position?) and again stable; it was unaffected by a (weak) tetanus that was just suprathreshold for $A$ firing at the start of the train but was markedly lowered by a tetanus that was strong enough to fire both $A$ and the LGs on every stimulus of the train. The effects of such strong trains on excitability of $A$ have been examined in 8 preparations (Fig. $8 B$ ); all but one showed at least mild potentiation, and the mean percentage drop (experimental minus control) was $36 \%$ at 30 min after the tetanus ( $p<0.01,2$-tailed Wilcoxon test).

\section{Insufficiency of paired pre- and postsynaptic firing}

Following the writings of Donald Hebb (1949), several investigators have considered the possibility that LTP induction might require strong stimuli during tetani because of their greater ability to recruit firing of the postsynaptic cells of the test pathway. Although it seems to us that the weight of evidence is against this (e.g., McNaughton et al., 1978), there remains some support in the literature (Scharfman and Sarvey, 1985). Figure $8 B$ shows data that bears on this issue. Eight preparations were tested for 
Figure 8. Effects of tetani on primary afferent-to-interneuron synapses in chronic preparations. Inset is a diagram indicating the synapses whose effectiveness was being assessed. Interneuron $A$ is the largest of a population of relatively phasic interneurons receiving input in only 1 segment; the other population of neurons indicated is more tonic, and each neuron receives input in a number of segments. $A$, Control session and experimental session illustrating the effect of a "weak" tetanus (pulse amplitudes just above interneuron A pretetanus threshold) and a "strong" tetanus (pulse amplitude sufficient to evoke LG spikes throughout train). $B$, Effects of tetani on the threshold of interneuron $A$ in 8 preparations. Tetanus pulse intensities sufficient to fire A but not LG $(100 \% \mathrm{~A})$ and $\mathrm{LG}$ ( $100 \%$ LG) throughout the tetanus are compared. The order in which these tetani were tested is indicated by the order of the bars.

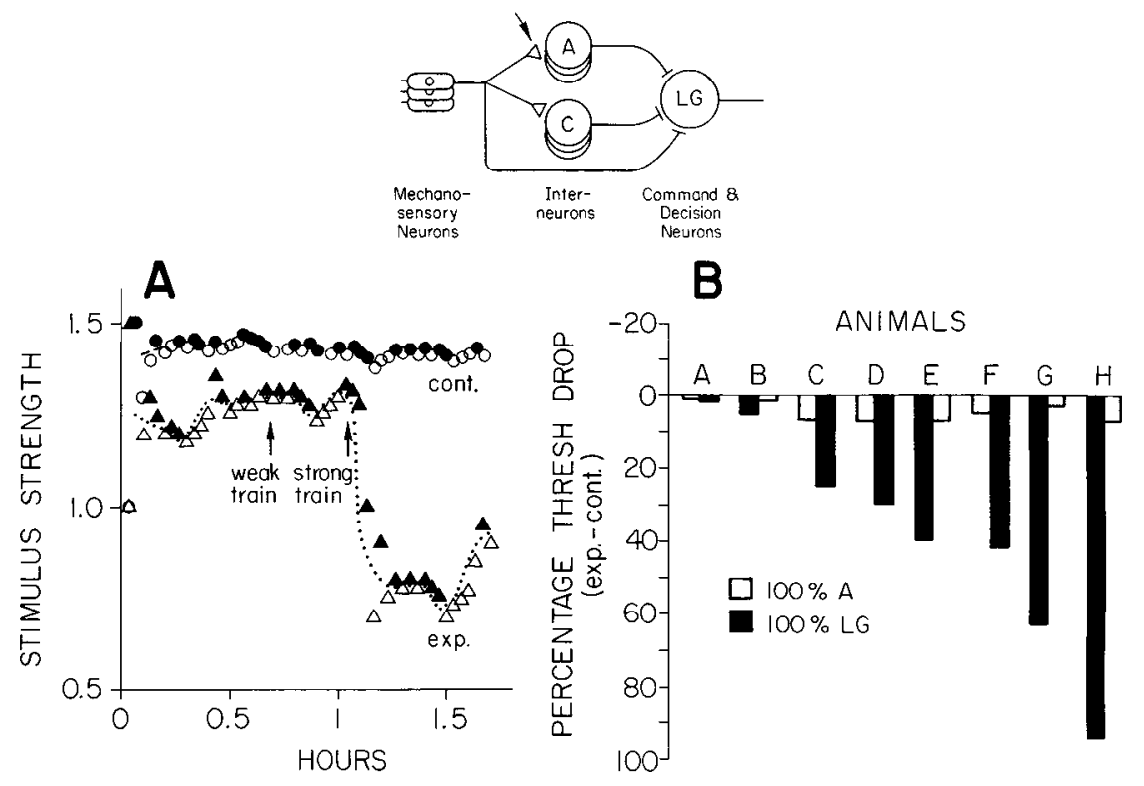

potentiation both with a tetanic stimulus that was strong enough to evoke $A$ firing throughout the tetanus and a stronger stimulus that was sufficiently strong to also evoke LG firing throughout the train. The weaker tetanus produced a mean threshold drop difference from control of $4.6 \%$ (very little potentiation), whereas the stronger produced a difference of $37.6 \%$. (These values differ significantly: $p<0.01,2$-tailed Wilcoxon test.)Thus, at this synapse a stimulus whose intensity is sufficient to cause postsynaptic firing throughout the tetanus is not necessarily sufficient to induce potentiation. While to our minds this reduces the likelihood that postsynaptic firing per se is important in the production of potentiation, it does not completely rule out a role for such firing because stronger stimuli almost certainly do cause more spikes per stimulus than weaker ones. We cannot confirm this directly in the present experiments, because the firing of the LGs and other large interneurons that it drives comes about the time that a second $A$ firing would occur and makes it impossible to detect $A$ firings in these chronic preparations. However, from other experiments we know that stimuli sufficient to fire the LGs usually cause 2-3 $A$ spikes, and we presume that this was also the case here.

\section{Stimulus specificity of potentiation}

We utilized the fact that, in the last abdominal ganglion, afferents contained in 5 separate nerve roots converge directly on interneuron $\mathrm{A}$ to evaluate the homosynapticity of potentiation of interneuron A responses. Because of the anatomical arrangement of the roots, it was convenient to place one stimulating electrode on roots 2, 3, and 4 and another on root 1 (Fig. 9, inset). The thresholds of $A$ responses to each stimulus were tracked in tandem by testing each electrode alternately (test trials at $1 / 2 \mathrm{~min}$; each root tested $1 / 4 \mathrm{~min}$ ). On one day, roots $2-4$ and, on another, root 1 was tetanized (each at $4 \mathrm{~Hz}$ with pulsc voltages several times pretetanus threshold). Figure 9 shows potentiation that is confined to the tetanized root. Of $10 \mathrm{ex}$ periments run, 5 were similar to that portrayed in showing no sign of transfer to untetanized roots, 4 showed slight and brief, but nevertheless clear transfer ( $<5 \%$ change of threshold), and one showed almost as much potentiation at the untetanized as the tetanized root. We cannot yet rule out the possibility that strong stimulus pulses during tetani fired some unintended afferents.

\section{Discussion}

\section{Relationship to other LTP phenomena}

The potentiation under study here is formally similar to hippocampal LTP. It is induced by relatively brief and modest tetani, it can persist for many hours, it is at least to some degree homosynaptic (see further below), and its induction requires the cooperative action of a number of afferents.

On the other hand, we have no evidence to suggest that the effect lasts beyond about $24 \mathrm{hr}$, so the apparent "permanence" that has been claimed for hippocampal LTP, and also for similarly named phenomena at some other synapses, may be lacking. Effects of elevated temperature on longevity, which may prolong LTP-like phenomena in mammals (Lynch and Baudry, 1984; Briggs et al., 1985), repeated induction, and other parametric variations remain to be examined. So do the effects of amines and peptides, some of which enhance transmitter release in this pathway (Glanzman and Krasne, 1983; Miller and Krasne, 1986) and seem to be able to induce long-lasting activity-dependent potentiation phenomena at other crustacean synapses (Breen and Atwood, 1983). Also required is study of interactions of potentiation-producing tetani with input from the facilitatory system that induces sensitization. We note, however, that although LTP is widely believed to be relatively permanent, the most thorough study available suggests that LTP in mammalian limbic forebrain at first decays with a time constant of about $1.5 \mathrm{hr}$ ("LIP1") and subsequently with one of about $5 \mathrm{~d}$ ("LTP2") (Racine et al., 1983). Decay of the slow component here was intermediatc betwecn thesc values.

Another feature that the potentiation under study here appears to share with LTP in other preparations is its caprice (Swanson et al., 1982). In the present instance this variability seems to be much greater for acute than for chronic preparations, and the difference is correlated with much greater ability of the reflex pathway to drive the LGs successfully throughout tetani 
Tetanize 2-4

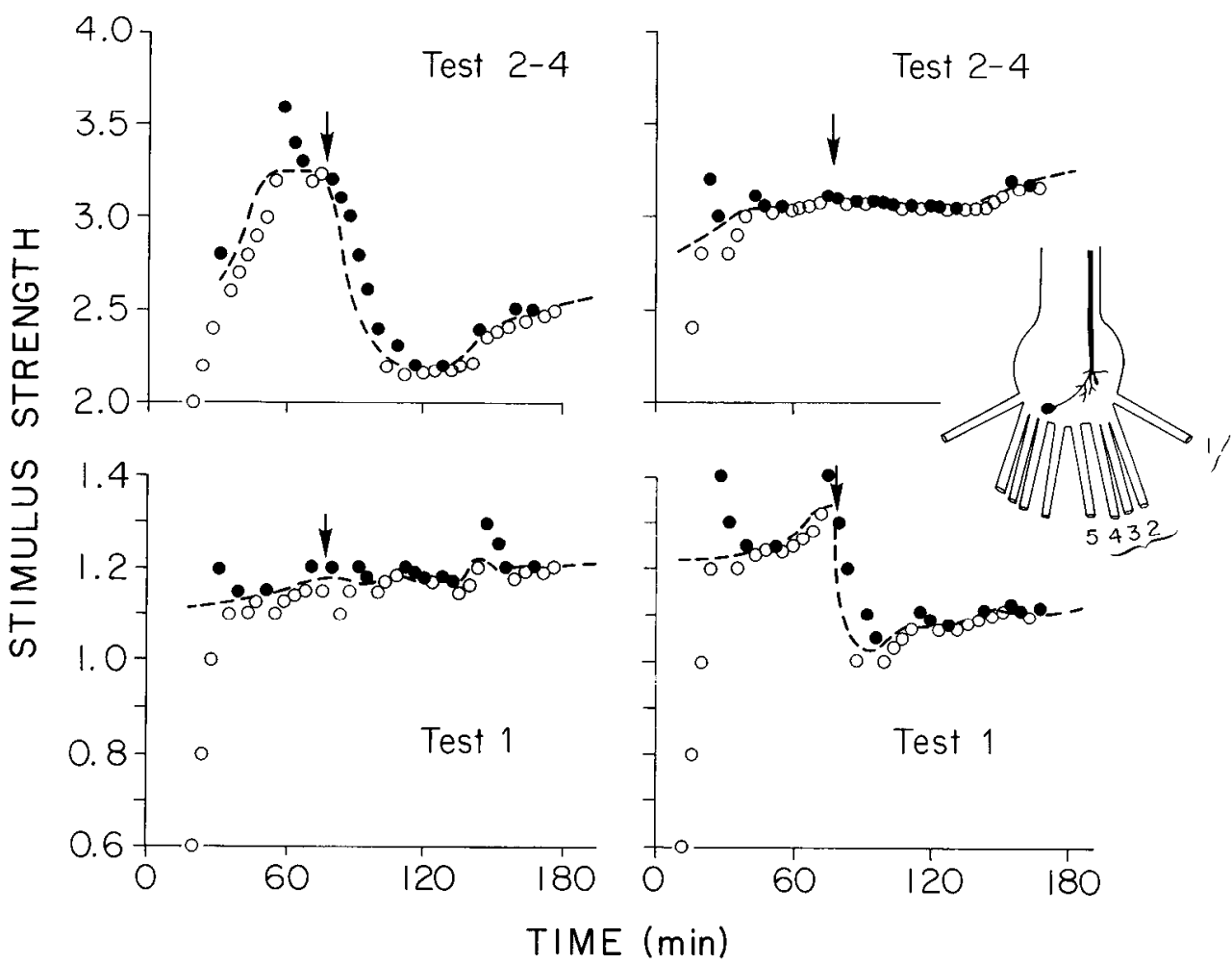

Figure 9. Homosynapticity of potentiation. Thresholds for fring interneuron A by stimulation of roots $2-4$ (above) and root 1 (below) were tracked concurrently and either roots $2-4$ (left) or root 1 (right) tetanized at the arrow. Inset, Diagram of the last ganglion with a cartoon of target interneuron $A$. in chronic preparations. However, LG firing is not itself either necessary or sufficient to induce potentiation, since comparable amounts of such firing produced by heterosegmental inputs did not cause potentiation, and in some preparations, both chronic and acute, clear potentiation occurred even when the LGs failed to fire during tetani. Conspicuous variables that warrant investigation are temperature (experiments on chronic preparations are done at about $21^{\circ} \mathrm{C}$, while those on acute preparations are done at about $17^{\circ} \mathrm{C}$ ), blood factors (Ringer's solution rather than blood bathes tissues in acute preparations), and changes of state that might be caused by dissection procedures in acute preparations.

The most interesting attribute of LTP as seen in both hippocampus and Aplysia, aside from longevity, is joint cooperativity and homosynapticity (and the closely related phenomenon of associativity); however, cooperativity has not yet been reported in the other systems showing long-lasting potentiations. The experiments reportcd herc provide clear evidence of cooperativity and at least some degrees of homosynapticity. However, evaluation of the precision of homosynapticity and direct tests of associativity are still needed.

We can envisage several scenarios to explain the cooperativity and observed degree of homosynapticity of this preparation.

1. Strong stimuli might recruit the diffusely distributing heterosynaptic facilitatory system that mediates sensitization, with homosynapticity arising from activity-dependent amplification of this facilitation. This is the explanation given for comparable phenomena in Aplysia (Hawkins et al., 1983; Walters and Byrne, 1983). However, as in Aplysia, strong stimuli cause widespread sensitization of escape reflex synapses even in the absence of activity of these synapses during the stimulation. Yet in the present experiments, transmission did not become enhanced outside the tetanized segment or even, in some experiments, at untetanized synapses within the tetanized hemiganglion. Furthermore, input to the facilitatory system is believed to occur only via rostral input points that were disconnected from abdominal circuitry in these experiments. Thus, it seems unlikely that the present phenomena involve the sensitization-producing system. It remains conceivable that despite cord severance the strong tetani causing potentiation are able to recruit a low level of facilitatory system activity that produces measurable facilitation only when amplified by target activity. However, preliminary experiments cause us to doubt that crayfish heterosynaptic facilitation is amplified by activity; a full cvaluation is planned.

2. Afferent activity might cause local release of a neurohumoral factor that promotes enhanced transmitter release or dendritic responsiveness at loci it reaches at sufficient concentration. The terminal fields of afferents in each root are spatially segregated from those of others. Thus, if the factor diffused, but modestly, this alone could account for the cooperativity and degree of homosynapticity demonstrated here. Release of the modulator might originate from the afferent terminals themselves, from an independent type of neuromodulatory cell, or from the terminals of the heterosynaptic facilitatory system in response to depolarization via local circuit input from afferents.

3. Strong excitatory input to dendrites of test pathway cells might promote induction of potentiation. The requirement of strong input (i.e., cooperativity) might reflect a need for intense dendritic depolarization (see, e.g., Kelso et al., 1986; Malinow and Miller, 1986) or a high local titer of some "second messenger" such as calcium ions (see, e.g., Lynch and Baudry, 1984; Collingridge, 1985). The degree of homosynapticity demonstrated here could result if afferents of different ganglionic roots terminated on different dendritic branches. Or, as proposed for hippocampal LTP (see, e.g., Sastry, 1982; Bliss and Dolphin, 1984; Collingridge, 1985; Sastry et al., 1986), the potentiation- 
promoting situation created by strong input might cause change only at active synapses (either presynaptically due to interaction of terminal activity with a retrograde signal provided by strongly excited dendrites or postsynaptically due to interactions of the potentiation-promoting state with some very local postsynaptic effect of test pathway input).

Interest in LTP-like phenomena arises especially because of the potential associative learning mechanisms they suggest. The mechanisms listed above, though all capable of explaining our observations, differ as to the associative properties they predict. Thus, the diffusely distributed activity-dependent facilitatory input envisaged in (1) could selectively increase the effectiveness with which afferents that were active at the time of facilitatory input could subsequently excite their targets, but unless one envisaged private facilitators for each target neuron, responses of arbitrarily chosen target neurons could not be selectively strengthened (see Krasne, 1984, for further discussion). Depending on the extent of diffusion of the locally released modulator postulated in (2), paired activity of a weakly stimulated and strongly stimulated input channel might or might not increase the potency of the weaker. If diffusion were slight, strong stimulation of 1 root might have little or no effect on synapses of others. If diffusion were more extensive, pairing would probably enhance responses to the weaker if activity in the weak channel and the facilitatory factor interacted synergistically. Even if effccts of the facilitatory factor were not inherently activitydependent, associative-like enhancements of transmission in the weak test pathway could occur if factor arriving by diffusion from the strongly stimulated input channel and locally released at terminals of the weaker channel combined to reach a concentration sufficient to cause change. (This would be especially likely if the factor's effect increased nonlinearly with concentration.) However, in this case, enhancements restricted to arbitrarily selected individual afferent fibers could probably not be achieved, and thus the mechanism probably could not provide a basis for real associative learning. Furthermore, if diffusion were great enough to permit interactions between activity of separate roots, it would probably also be too great to affect synapses on specific postsynaptic cells selectively, which would again limit its value as a substrate for true learning in higher animals. Hypothesis (3) might or might not predict associativity, depending on the particular versions considered. If homosynapticity were simply due to spatial segregation on dendritic arbors of inputs from different roots, then as with the diffusion of an extracellular factor, associative properties might be absent or limited, depending on extent of intracellular spread and other factors. If, on the other hand, the postsynaptic effects of strong input were widespread and homosynapticity were conferred by dependence on presynaptic activity or on well-localized postsynaptic events, associativity would be expected and could be highly specific to synapses between particular pre- and postsynaptic neurons.

Further tests of the precision of homosynapticity and associativity will thus help in beginning to discriminate the above hypotheses, as well as defining the information-processing capabilities of the mechanisms at work here.

This discussion of associative properties should be qualified by recognition that the functional capabilities of a cellular mechanism may depend on the context in which it is utilized. For example, if the LTP seen here were induced by a large influx of $\mathrm{Ca}^{2+}$ postsynaptically at active synapses on strongly depolarized dendritic membrane [a currently popular hypothesis for hip- pocampal LTP (Lynch and Baudry, 1984; Collingridge, 1985)], the increases might well not be fully homosynaptic because the $\mathrm{Ca}^{2+}$ would probably diffuse somewhat along the dendrite and thus cause alteration of immediately adjacent synapses that were not active. Yet, in hippocampus the same mechanism might yield perfect specificity because the necks of the spines on which each afferent terminates would greatly reduce diffusion between different postsynaptic active zones. Thus, the same basic mechanism could be operating here and in the hippocampus, yet the phenomena of potentiation might differ in significant ways. Indeed, it is possible that in invertebrates full homosynapticity of activity-dependent changes of synaptic transmission can only be achieved by localizing change presynaptically, whereas the compartmentalization provided by dendritic spines may make appropriate postsynaptic neurons useful sites of change in vertebrates.

\section{Behavioral significance}

The behavioral consequence of the phenomenon described here should be an increased probability of escape from stimuli applied to bodily locations that were previously stimulated vigorously. In the intact animal, segmentally and root-specific potentiation is complemented by a body-wide sensitization of the escape response following traumatic stimulation. Perhaps the locally specific potentiation is an evolutionary progenitor of the suprasegmental sensitization, which is now the organism's main means of increasing the sensitivity of its escape reaction in times of danger. Alternatively, the locally specific potentiation described here might allow a useful local augmentation of sensitization. One might suppose, for example, that if a predator caught a crayfish by the tailtan (and the crayfish managed to get away), then thereafter the animal would be more likely to escape from any stimulus but might be especially sensitive to mechanical disturbances in the region of its tailfan. A slightly different function for a greater increase of excitability at the site of a traumatic stimulus than elsewhere relates to the habituation to which the escape reflex is prone (Krasne, 1969; Wine et al., 1975). Habituation results from a homosynaptic depression of transmission at previously active primary afferent-to-interneuron synapses (Zucker, 1972; Krasne, 1976). If a traumatic stimulus is to create as great escape reaction excitability for stimuli in the sensory field of prior traumatic stimulation as elsewhere, then an additional increment of enhancement may be needed in the traumatically stimulated field to compensate for depression caused there by the traumatic stimulus; a cooperative homosynaptic potentiation could achieve such compensation. The activity-dependent amplification of heterosynaptic facilitation found in Aplysia (Hawkins et al., 1983; Walters and Byrne, 1983) could have a similar natural function, with the very interesting and suggestive enhancements of facilitation producible at other bodily sites stimulated during trauma being a fortuitous consequence of the way that such compensation is achieved. Of course, the mechanism might still be used for creating arbitrary associations in higher animals.

It is also possible that the susceptibility of the afferent-tointerneuron synapses of the LG circuit to LTP is of little or no behavioral relevance but, rather, reflects the presence of cellular mechanisms used during development. It has been suggested on several occasions that synapses whose presynaptic terminals are active when their target cells are strongly driven by other inputs are stabilized (or at least preserved), while synapses whose terminals are inactive during strong postsynaptic driving are weak- 
ened or destroyed during development (see, e.g., Wiesel and Hubel, 1965; Stent, 1973; Sanes and Constantine-Paton, 1985). Formally, this developmental phenomenon is very similar to associative LTP, and the mechanisms responsible for the developmental phenomenon, operating vestigially in the adult, could conceivably be responsible for LTP-like phenomena. Thus, it is perhaps relevant that driving of interneuron $A$ by its normal innervation has recently been found to reduce the extent to which regenerating sensory neurons will form synapses on the interneuron (Krasne, 1987).

\section{LTP at electrical synapses?}

Although potentiation is conspicuous at the chemical synapses between primary afferents and interneuron $A$, and will also presumably be found to occur at other afferent-to-first-order interneuron synapses, some potentiation occasionally seemed to occur at the supposedly electrical synapses that primary afferents make directly on LG dendrites. Recent evidence suggests that electrical junctions are more malleable than once believed (Giaume and Korn, 1984; Piccolino et al., 1984), and the present observations might be an instance of electrical synapse plasticity. However, ultrastructurally the synapses of afferents on the LGs, like many other "electrical" synapses, show, in addition to gap junctions, morphological specializations of a type usually considered to indicate chemical synapses (S. C. Lee and F. B. Krasne, unpublished observations); thus, potentiation at these synapses might be contributed by a chemically mediated component of the transmission process.

\section{References}

Anderson, P., S. H. Sundberg, O. Sveen, and H. Wigström (1977) Specific long-lasting potentiation of synaptic transmission in hippocampal slices. Nature 266: 736-737.

Barrionuevo, G., and T. H. Brown (1983) Associative long-term potentiation in hippocampal slices. Proc. Natl. Acad. Sci. USA 80:73477351.

Baxter, D. A., G. D. Bittner, and T. H. Brown (1985) Quantal mechanism of long-term synaptic potentiation. Proc. Natl. Acad. Sci. USA 82: 5978 .

Bliss, T. V. P., and A. C. Dolphin (1984) Where is the locus of longterm potentiation? In Neurobiology of Learning and Memory, G. Lynch, J. McGaugh, and N. Weinberger, eds., pp. 451-458, Guilford, New York.

Bliss, T. V. P., and A. Gardner-Medwin (1973) Long-lasting potentiation of synaptic transmission in the dentate area of unanesthetized rabbit following stimulation of the perforant path. J. Physiol. (Lond.) 232: 357-374.

Bliss, T. V. P., and T. Lomo (1973) Long-lasting potentiation of synaptic transmission in the dentate area of the anaesthetized rabbit following stimulation of the perforant path. J. Physiol. (Lond.) 232 : 331-356.

Breen, C. A., and H. L. Atwood (1983) Octopamine-A neurohormone with presynaptic activity-dependent effects at crayfish neuromuscular junction. Nature 303: 716-718.

Briggs, C. A., T. H. Brown, and D. A. McAfee (1985) Neurophysiology and pharmacology of long-term potentiation in the rat sympathetic ganglion. J. Physiol. (Lond.) 359: 503-521.

Bryan, J. S., and F. B. Krasne (1977) Presynaptic inhibition: The mechanism of protection from habituation of the crayfish lateral giant fibre escape response. J. Physiol. (Lond.) 271: 369-390.

Chang, F. L. F., and W. T. Greenough (1984) Transient and enduring morphological correlates of synaptic activity and efficacy change in the rat hippocampal slice. Brain Res. 309: 35-46.

Collingridge, G. I. (1985) Long term potentiation in the hippocampus: Mechanisms of initiation and modulation by neurotransmitters. Trends Pharmacol. Sci. 6: 407-411.

Douglas, R. M., and G. V. Goddard (1975) Long-term potentiation of the perforant path-granule cell synapse in the rat hippocampus. Brain Res. 86: 205-215.
Dunwiddie, T., and G. Lynch (1978) Long-term potentiation and depression of synaptic responses in the rat hippocampus: Localization and frequency dependency. J. Physiol. (Lond.) 276: 353-367.

Giaume, C., and H. Korn (1984) Voltage-dependent dye coupling at a rectifying electrotonic synapse of the crayfish. J. Physiol. (Lond.) 356: $151-167$.

Glanzman, D. L., and F. B. Krasne (1983) Serotonin and octopamine have opposite modulatory effects on the crayfish's lateral giant escape reaction. J. Neurosci. 3: 2263-2269.

Hawkins, R. D., T. W. Abrams, T. J. Carew, and E. R. Kandel (1983) A cellular mechanism of classical conditioning in Aplysia: Activitydependent amplification of presynaptic facilitation. Science 219:400405.

Hebb, D. O. (1949) The Organization of Behavior, Wiley-Interscience, New York.

Hopkins, W. F., and D. Johnston (1984) Frequency-dependent noradrenergic regulation of long-term potentiation in hippocampus. Science 226: $350-352$.

Kelso, S. R., and T. H. Brown (1986) Differential conditioning of associative synaptic enhancement in hippocampal brain slices. Science 232: 85-87.

Kelso, S. R., A. H. Ganong, and T. H. Brown (1986) Hebbian synapses in hippocampus. Proc. Natl. Acad. Sci. USA 83: 5326-5330.

Kennedy, D., R. L. Calabrese, and J. J. Wine (1974) Presynaptic inhibition: Primary afferent depolarization in crayfish neurons. Science 186: 451-454.

Krasne, F. B. (1969) Excitation and habituation of the crayfish escape reflex: The depolarizing response in lateral giant fibres of the isolated abdomen. J. Exp. Biol. 50: 29-46.

Krasne, F. B. (1976) Invertebrate systems as a means of gaining insight into the nature of learning and memory. In Neural Mechanisms of Learning and Memory, M. R. Rosenzweig and E. L. Bennett, eds., pp. 401-429, MIT Press, Cambridge, MA.

Krasne, F. B. (1984) Physiological analysis of learning in invertebrates. In Cortical Integration: Basic, Archicortical and Cortical Association Levels of Neural Integration, F. Reinoso-Suarez and C. Ajmone-Marsan, eds., pp. 53-76, Raven Press, New York.

Krasne, F. B. (1987) Silencing normal input permits regenerating foreign afferents to innervate an identified crayfish sensory interneuron. J. Neurobiol. 18: 61-73.

Krasne, F. B., and D. L. Glanzman (1986) Sensitization of the crayfish lateral giant escape reaction. J. Neurosci. 6:1013-1020.

Lee, K. S., F. Schottler, M. Oliver, and G. Lynch (1980) Brief bursts of high-frequency stimulation produce two types of structural change in rat hippocampus. J. Neurophysiol. 44: 247-258.

Levy, W. B., and O. Steward (1983) Temporal contiguity requirement for long-term associative potentiation/depression in the hippocampus. Neuroscience 8: 791-797.

Lynch, G., and M. Baudry (1984) The biochemistry of memory: A new and specific hypothesis. Science 224: 1057-1063.

Malinow, R., and J. P. Miller (1986) Postsynaptic hyperpolarization during conditioning reversibly blocks induction of long-term potentiation. Nature 320: 529-530.

McNaughton, B. L., R. M. Douglas, and G. V. Goddard (1978) Synaptic enhancement in fascia dentata: Cooperativity among co-active afferents. Brain Res. 157: 277-293.

Miller, M. W., and F. B. Krasne (1986) Proctolin modulates sensory neurotransmission in the crayfish lateral giant escape reflex circuitry. Soc. Neurosci. Abstr. 12: 243.

Piccolino, M., J. Neyton, and H. M. Gerschenfeld (1984) Decrease of gap junction permeability induced by dopamine and cyclic adenosine $3^{\prime}: 5^{\prime}$-monophosphate in horizontal cells of turtle retina. J. Neurosci. 4: $2477-2488$.

Racine, R. J., N. W. Milgran, and S. Hafner (1983) Long-term potentiation phenomena in the rat limbic forebrain. Brain Res. 260: 217-231.

Sanes, D. H., and M. Constantine-Paton (1985) The sharpening of frequency tuning curves requires patterned activity during development in the mouse, Mus musculus. J. Neurosci. 5: 1152-1166.

Sastry, B. R. (1982) Presynaptic change associated with long-term potentiation in hippocampus. Life Sci. 30: 2003-2008.

Sastry, B. R., J. W. Goh, and A. Auyeung (1986) Associative induction of posttetanic and long-term potentiation in CAl neurons of rat hippocampus. Science 232: 988-990.

Scharfman, H. E., and J. M. Sarvey (1985) Postsynaptic firing during 
repetitive stimulation is required for long-term potentiation in hippocampus. Brain Res. 331: 267-274.

Stent, G. (1973) A physiological mechanism for Hebb's postulate of learning. Proc. Natl. Acad. Sci. USA 70:997-1001.

Swanson, L. W., T. J. Teyler, and R. F. Thompson (1982) Hippocampal long-term potentiation: Mechanisms and implications for memory. Neurosci. Res. Prog. Bull. 20: 613-769.

van Harreveld, A. (1936) A physiological solution for freshwater crustaceans. Proc. Soc. Exp. Biol. Med. 34: 428-432.

Walters, E. T., and J. H. Byrne (1983) Slow depolarization produced by associative conditioning of Aplysia sensory neurons may enhance $\mathrm{Ca}^{2+}$ cntry. Brain Res. 280: 165-168.

Walters, E. T., and J. H. Byrne (1985) Long-term enhancement produced by activity-dependent modulation of Aplysia sensory neurons. J. Neurosci. 5: 662-672.
Wiesel, T., and D. Hubel (1965) Comparison of monocular and binocular deprivation in cortex of cats. J. Neurophysiol. 28: 1029-1040.

Wine, J. J., and F. B. Krasne (1982) The cellular organization of crayfish escape behavior. In The Biology of Crustacea, vol. 4, D. C. Sandeman and H. L. Atwood, eds., pp. 241-292, Academic Press, New York.

Wine, J. J., F. B. Krasne, and L. Chen (1975) Habituation and inhibition of the crayfish lateral giant fibre escape response. J. Exp. Biol. 62: 771-782.

Zucker, R. S. (1972) Crayfish escape behavior and central synapses. II. Physiological mechanisms underlying behavioral habituation. J. Neurophysiol. 35: 621-637. 